\title{
On Computation of Recently Defined Degree-Based Topological Indices of Some Families of Convex Polytopes via M-Polynomial
}

\author{
Deeba Afzal $\left(\mathbb{D},{ }^{1}\right.$ Farkhanda Afzal ${ }^{D},{ }^{2}$ Mohammad Reza Farahani ${ }^{\circ},{ }^{3}$ and Samia Ali ${ }^{1}$ \\ ${ }^{1}$ Department of Mathematics and Statistics, The University of Lahore, Lahore 54000, Pakistan \\ ${ }^{2}$ MCS, National University of Sciences and Technology, Islamabad, Pakistan \\ ${ }^{3}$ Department of Applied Mathematics, Iran University of Science and Technology (IUST), Narmak, Tehran 16844, Iran
}

Correspondence should be addressed to Mohammad Reza Farahani; mrfarahani88@gmail.com

Received 12 May 2021; Accepted 17 June 2021; Published 8 July 2021

Academic Editor: Muhammad Javaid

Copyright ( $\odot 2021$ Deeba Afzal et al. This is an open access article distributed under the Creative Commons Attribution License, which permits unrestricted use, distribution, and reproduction in any medium, provided the original work is properly cited.

Topological indices are of incredible significance in the field of graph theory. Convex polytopes play a significant role both in various branches of mathematics and also in applied areas, most notably in linear programming. We have calculated some topological indices such as atom-bond connectivity index, geometric arithmetic index, $K$-Banhatti indices, and $K$-hyper-Banhatti indices and modified $K$-Banhatti indices from some families of convex polytopes through $M$-polynomials. The $M$-polynomials of the graphs provide us with a great help to calculate the topological indices of different structures.

\section{Introduction}

Graph theory is a powerful and definable field of mathematics that in every field of science has countless adaptations. In graph theory, different characteristics of graphs are defined [1]. The topological indices display the graphical structure and many other characteristics in graphs. They are typically based on the distances between the vertices, on vertex degrees, or on the graph depicted by the matrix.

Using the general polynomial is the general method by which we can generate the unique form of topological indices. Hosoya polynomial is an example of this form [2]. Similarly $M$-polynomial [3] that is an algebraic polynomial can also explain the behavior of the molecular structure. It is also graph representative mathematical object which is the most general polynomial developed till now and gives us the formulas that are very close to the degree-based topological indices.

The $M$-polynomial introduced in 2015 for a graph $G$ by Emeric Deutsch and Sandi Klavžar [4] is defined as

$$
M(G, x, y)=\sum_{\delta \leq i \leq j \leq \Delta} m_{i j}(G) x^{i} y^{j} .
$$

Here, $m_{i j}(G)$ is the number of edges $u v \in E(G)$, such that $\left\{d_{v}, d_{u}\right\}=\{i, j\}, \quad \delta=\min \left\{d_{v} \mid v \in V(G)\right\}, \quad$ and $\Delta=$ $\max \left\{d_{v} \mid v \in V(G)\right\}$.

We generally find topological indices using the definitions, but we can also find them using the chosen graph's derivatives and integrals on $M$-polynomials. So if we have an $M$-polynomial of the graph, we can measure its various topological indices. Several $M$-polynomials of several graphs have been added over the last few years $[3,5,6]$.

In 1947, first index, the wiener index suggested by Wiener. A well-known degree-based topological invariant known as atom-bond connectivity index [7] was purposed in 1998, by Estrada et al. This index is denoted by ABC. Damir Vukičević and Boris Furtula suggested a new index in 2009, known as geometric arithmetic index [8] which is denoted by GA. $K$-Banhatti and $K$-hyper-Banhatti indices were introduced by Kulli in 2016 [9, 10]. Similarly, in 2017, Kulli introduced some more important topological indices [11] named as modified $K$-Banhatti indices. Here we will use the operators which are defined as 


$$
\begin{aligned}
& D_{x} g(x, y)=x \frac{\partial(g(x, y))}{\partial x} \\
& D_{y} g(x, y)=y \frac{\partial(g(x, y))}{\partial y} \\
& L_{x} g(x, y)=g\left(x^{2}, y\right), \\
& L_{y} g(x, y)=g\left(x, y^{2}\right), \\
& S_{x} g(x, y)=\int_{0}^{x} \frac{g(t, y)}{t} \mathrm{~d} t, \\
& S_{y} g(x, y)=\int_{0}^{y} \frac{g(x, t)}{t} \mathrm{~d} t, \\
& J g(x, y)=g(x, x), \\
& Q_{\alpha} g(x, y)=x^{\alpha} g(x, y), \\
& D_{x}^{1 / 2} g(x, y)=\sqrt{x \frac{\partial(g(x, y))}{\partial x}} \cdot \sqrt{g(x, y)}, \\
& D_{y}^{1 / 2} g(x, y)=\sqrt{y \frac{\partial(g(x, y))}{\partial y}} \cdot \sqrt{g(x, y)}, \\
& S_{x}^{1 / 2} g(x, y)=\sqrt{\int_{0}^{x} \frac{g(t, y)}{t} \mathrm{~d} t} \cdot \sqrt{g(x, y)}, \\
& S_{y}^{1 / 2} g(x, y)=\sqrt{\int_{0}^{y} \frac{g(x, t)}{t} \mathrm{~d} t} \cdot \sqrt{g(x, y)} .
\end{aligned}
$$

Now we will calculate closed forms of many degreebased topological indices of some families of convex polytopes by using $M$-polynomials.

\section{Some Families of Convex Polytopes and Their Computational Aspects}

If we add new edges in the graph of convex polytopes $Q_{n}$, then we get a graph named as convex polytopes $T_{n}$. It will consist of three-sided face, five-sided face, and $n$-sided faces. $a_{i+1} b_{i}$, i.e., $V\left(T_{n}\right)=V\left(Q_{n}\right)$ and $V\left(T_{n}\right)$ and $V\left(Q_{n}\right) \cup\left\{a_{i+1} b_{i} \mid l \leq i \leq n\right\}$ (for example, see Figure 1).

Similarly, the graph of convex polytope $A_{n}$, i.e., double antiprism, can be acquired from the graph of convex polytopes $R_{n}$ by adding new edges $b_{i+1} c_{i}$, i.e., $V\left(A_{n}\right)=V\left(R_{n}\right)$ and $V\left(A_{n}\right)=\left(R_{n}\right) \cup\left\{b_{i=l} c_{i} \mid l \leq i \leq n\right\} \quad$ (for example, see Figure 2).

Convex polytopes (double antiprism) $S_{n}$ graph can be obtained from the graph of convex poly tope $Q_{n}$ by adding new edges $\quad c_{i} c_{i+1}$, i.e., $V\left(S_{n}\right)=V\left(Q_{n}\right) \quad$ and $V\left(S_{n}\right)=V\left(Q_{n}\right) \cup\left\{c_{i} c_{i+1} \mid l \leq i \leq n\right\} \quad$ (for example, see Figure 3).

\section{M-Polynomial and Topological Indices of Convex Polytopes $T_{n}$}

Now we will find the topological indices of $T_{n}$ using its $M$ polynomial.

Theorem 1. The M-polynomial of $T_{n}$ is $M\left[T_{n} ; x ; y\right]$ $=2 n x^{3} y^{3}+2 n x^{3} y^{6}+n x^{4} y^{4}+2 n x^{4} y^{6}+n x^{6} y^{6}$. Then

(1) $A B C\left[T_{n}\right]=(n / 12)(16+4 \sqrt{14}+3 \sqrt{6}+\sqrt{48}+\sqrt{10})$

(2) $G A\left[T_{n}\right]=(n / 15)(30+10 \sqrt{2}+6 \sqrt{6})$

(3) $B_{1}\left[T_{n}\right]=178 n$

(4) $B_{2}\left[T_{n}\right]=502 n$

(5) $H B_{1}\left[T_{n}\right]=2126 n$

(6) $H B_{2}\left[T_{n}\right]=2518 n$

(7) ${ }^{m} B_{1}\left[T_{n}\right]=(17033 / 10920) n$

(8) ${ }^{m} B_{2}\left[T_{n}\right]=(1171 / 1680) n$

(9) $H_{b}\left[T_{n}\right]=(17033 / 5460) n$.

Proof

$$
\begin{gathered}
D_{x} g(x ; y)=6 n x^{3} y^{3}+6 n x^{3} y^{6}+4 n x^{4} y^{4}+8 n x^{4} y^{6}+6 n x^{6} y^{6} \\
D_{y} g(x ; y)=6 n x^{3} y^{3}+12 n x^{3} y^{6}+4 n x^{4} y^{4}+12 n x^{4} y^{6}+6 n x^{6} y^{6} \\
\left(D_{x}+D_{y}\right)[g(x ; y)]=12 n x^{3} y^{3}+18 n x^{3} y^{6}+8 n x^{4} y^{4}+20 n x^{4} y^{6}+12 n x^{6} y^{6} \\
S_{y}^{1 / 2} g(x, y)=\frac{2}{\sqrt{3}} n x^{3} y^{3}+\frac{2}{\sqrt{6}} n x^{3} y^{6}+\frac{n}{2} x^{4} y^{4}+\frac{2}{\sqrt{6}} n x^{4} y^{6}+\frac{n}{\sqrt{2}} x^{6} y^{6}, \\
S_{x}^{1 / 2} S_{y}^{1 / 2} g(x, y)=\frac{2}{3} n x^{3} y^{3}+\frac{2}{3 \sqrt{2}} n x^{3} y^{6}+\frac{n}{4} x^{4} y^{4}+\frac{1}{\sqrt{6}} n x^{4} y^{6}+\frac{n}{6} x^{6} y^{6},
\end{gathered}
$$




$$
\begin{aligned}
& J S_{x}^{1 / 2} S_{y}^{1 / 2} g(x, y)=\frac{2}{3} n x^{6}+\frac{2}{3 \sqrt{2}} n x^{9}+\frac{n}{4} x^{8}+\frac{1}{\sqrt{6}} n x^{10}+\frac{n}{6} x^{12}, \\
& Q_{-2} J S_{x}^{1 / 2} S_{y}^{1 / 2} g(x, y)=\frac{2}{3} n x^{4}+\frac{2}{3 \sqrt{2}} n x^{7}+\frac{n}{4} x^{6}+\frac{1}{\sqrt{6}} n x^{8}+\frac{n}{6} x^{10}, \\
& D_{x}^{1 / 2} Q_{-2} J S_{x}^{1 / 2} S_{y}^{1 / 2} g(x, y)=\frac{4}{3} n x^{4}+\frac{\sqrt{14}}{3} n x^{7}+\frac{\sqrt{6}}{4} n x^{6}+\frac{\sqrt{8}}{\sqrt{6}} n x^{8}+\frac{\sqrt{10}}{6} n x^{10}, \\
& D_{y}^{1 / 2}[g(x, y)]=2 \sqrt{3} n x^{3} y^{3}+2 \sqrt{6} n x^{3} y^{6}+2 n x^{4} y^{4}+2 \sqrt{6} n x^{4} y^{6}+n \sqrt{6} x^{6} y^{6}, \\
& D_{x}^{1 / 2} D_{y}^{1 / 2}[g(x, y)]=6 n x^{3} y^{3}+2 \sqrt{6} \sqrt{3} n x^{3} y^{6}+4 n x^{4} y^{4}+2 \sqrt{4} \sqrt{6} n x^{4} y^{6}+6 n x^{6} y^{6} \text {, } \\
& J D_{x}^{1 / 2} D_{y}^{1 / 2}[g(x, y)]=6 n x^{6}+2 \sqrt{6} \sqrt{3} n x^{9}+4 n x^{8}+2 \sqrt{4} \sqrt{6} n x^{10}+6 n x^{12}, \\
& S_{x} J D_{x}^{1 / 2} D_{y}^{1 / 2}[g(x, y)]=n x^{6}+\frac{2}{3} \sqrt{2} n x^{9}+\frac{1}{2} n x^{8}+\frac{2}{5} \sqrt{6} n x^{10}+\frac{1}{2} n x^{12}, \\
& 2 S_{x} J D_{x}^{1 / 2} D_{y}^{1 / 2}[g(x, y)]=2 n x^{6}+\frac{4}{3} \sqrt{2} n x^{9}+n x^{8}+\frac{4}{5} \sqrt{6} n x^{10}+n x^{12}, \\
& J g(x ; y)=2 n x^{6}+n x^{8}+2 n x^{9}+2 n x^{10}+n x^{12} \\
& Q_{-2} J g(x ; y)=2 n x^{4}+n x^{6}+2 n x^{7}+2 n x^{8}+n x^{10} ; \\
& D_{x} Q_{-2} J g(x ; y)=8 n x^{4}+6 n x^{6}+14 n x^{7}+16 n x^{8}+10 n x^{10} ; \\
& 2 D_{x} Q_{-2} J g(x ; y)=16 n x^{4}+12 n x^{6}+28 n x^{7}+32 n x^{8}+20 n x^{10} ; \\
& J\left(D_{x}+D_{y}\right) g(x, y)=12 n x^{6}+18 n x^{9}+8 n x^{8}+20 n x^{10}+12 n x^{12} \text {; } \\
& Q_{-2} J\left(D_{x}+D_{y}\right) g(x ; y)=12 n x^{4}+18 n x^{7}+8 n x^{6}+20 n x^{8}+12 n x^{10} \\
& D_{x} Q_{-2} J\left(D_{x}+D_{y}\right) g(x ; y)=48 n x^{4}+126 n x^{7}+48 n x^{6}+160 n x^{8}+120 n x^{10} \text {; } \\
& D_{x}^{2} g(x ; y)=18 n x^{3} y^{3}+18 n x^{3} y^{6}+16 n x^{4} y^{4}+32 n x^{4} y^{6}+36 n x^{6} y^{6} \\
& D_{y}^{2} g(x ; y)=18 n x^{3} y^{3}+72 n x^{3} y^{6}+16 n x^{4} y^{4}+72 n x^{4} y^{6}+36 n x^{6} y^{6} \\
& D_{x}^{2} Q_{-2} J g(x ; y)=32 n x^{4}+98 n x^{7}+36 n x^{6}+128 n x^{8}+100 n x^{10} ; \\
& 2 D^{2} Q_{-2} J g(x ; y)=64 n x^{4}+196 n x^{7}+72 n x^{6}+256 n x^{8}+200 n x^{10} ; \\
& 2 D_{x} Q_{-2} J\left(D_{x}+D_{y}\right) g(x ; y)=96 n x^{4}+252 n x^{7}+96 n x^{6}+320 n x^{8}+240 n x^{10} \text {; } \\
& \left(D_{x}^{2}+D_{y}^{2}\right) g(x ; y)=36 n x^{3} y^{3}+90 n x^{3} y^{6}+32 n x^{4} y^{4}+104 n x^{4} y^{6}+72 n x^{6} y^{6} \\
& J\left(D_{x}^{2}+D_{y}^{2}\right) g(x ; y)=36 n x^{6}+90 n x^{9}+32 n x^{8}+104 n x^{10}+72 n x^{12} \\
& Q_{-2} J\left(D_{x}^{2}+D_{y}^{2}\right) g(x ; y)=36 n x^{4}+90 n x^{7}+32 n x^{6}+104 n x^{8}+72 n x^{10} \text {; } \\
& D_{x}^{2} Q_{-2} J\left(D_{x}^{2}+D_{y}^{2}\right) g(x ; y)=576 n x^{4}+4410 n x^{7}+1152 n x^{6}+6656 n x^{8}+7200 n x^{10} ; \\
& L_{x} g(x ; y)=2 n x^{6} y^{3}+2 n x^{6} y^{6}+n x^{8} y^{4}+2 n x^{8} y^{6}+n x^{12} y^{6} \\
& L_{y} g(x ; y)=2 n x^{3} y^{6}+2 n x^{3} y^{12}+n x^{4} y^{8}+2 n x^{4} y^{12}+n x^{6} y^{12} \\
& J\left(L_{x}+L_{y}\right) g(x ; y)=4 n x^{9}+4 n x^{12}+2 n x^{14}+2 n x^{15}+2 n x^{16}+2 n x^{18} ; \\
& Q_{-2} J\left(L_{x}+L_{y}\right) g(x ; y)=4 n x^{7}+4 n x^{10}+2 n x^{12}+2 n x^{13}+2 n x^{14}+2 n x^{16} \\
& S_{x} Q_{-2} J\left(L_{x}+L_{y}\right) g(x, y)=\frac{4}{7} n x^{7}+\frac{4}{10} n x^{10}+\frac{1}{6} n x^{12}+\frac{2}{13} n x^{13}+\frac{1}{7} n x^{14}+\frac{1}{8} n x^{16} \text {, } \\
& S_{y} g(x, y)=\frac{2}{3} n x^{3} y^{3}+\frac{1}{3} n x^{3} y^{6}+\frac{1}{4} n x^{4} y^{4}+\frac{1}{3} n x^{4} y^{6}+\frac{1}{6} n x^{6} y^{6}, \\
& S_{x} g(x, y)=\frac{2}{3} n x^{3} y^{3}+\frac{2}{3} n x^{3} y^{6}+\frac{1}{4} n x^{4} y^{4}+\frac{1}{2} n x^{4} y^{6}+\frac{1}{6} n x^{6} y^{6}, \\
& \left(S_{x}+S_{y}\right) g(x, y)=\frac{4}{3} n x^{3} y^{3}+n x^{3} y^{6}+\frac{1}{2} n x^{4} y^{4}+\frac{5}{6} n x^{4} y^{6}+\frac{1}{3} n x^{6} y^{6} \text {, }
\end{aligned}
$$




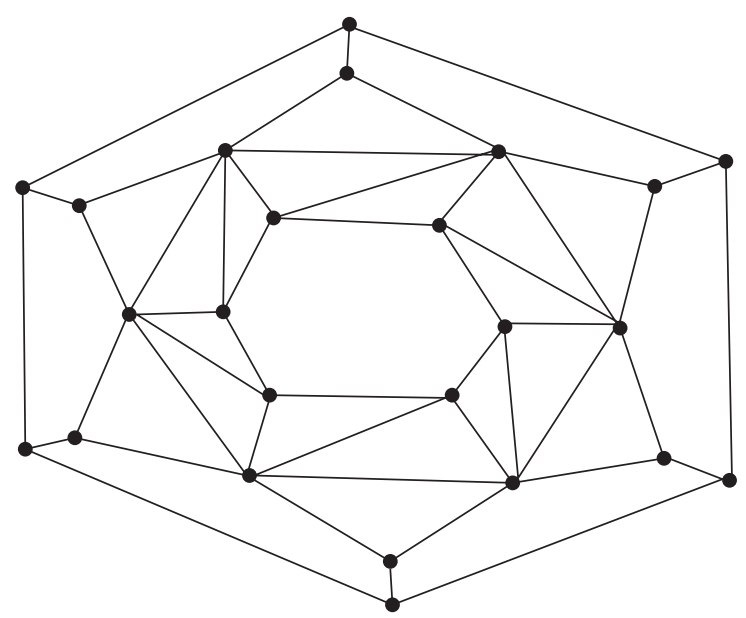

FIgURE 1: Graph of convex polytope $T_{6}$.

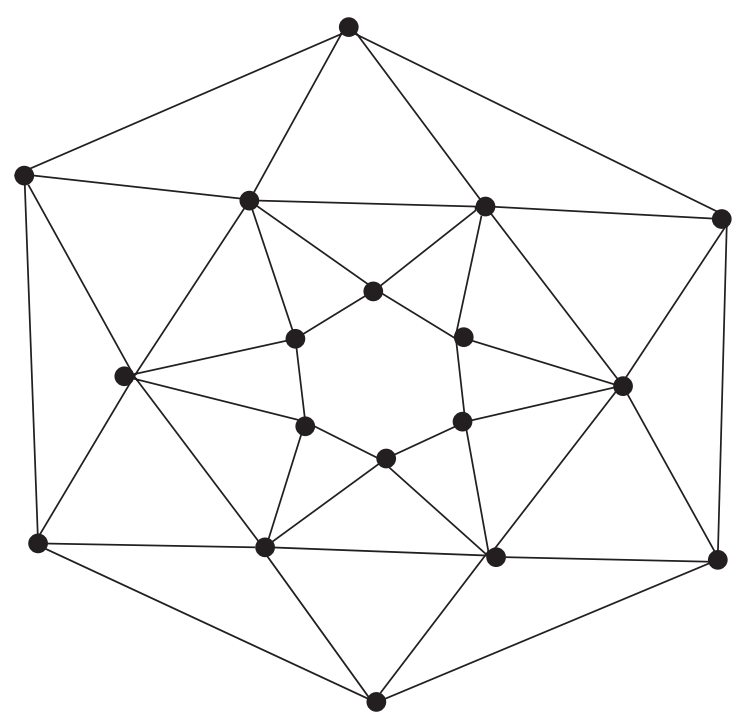

FIgURe 2: Graph of double antiprism $A_{6}$.

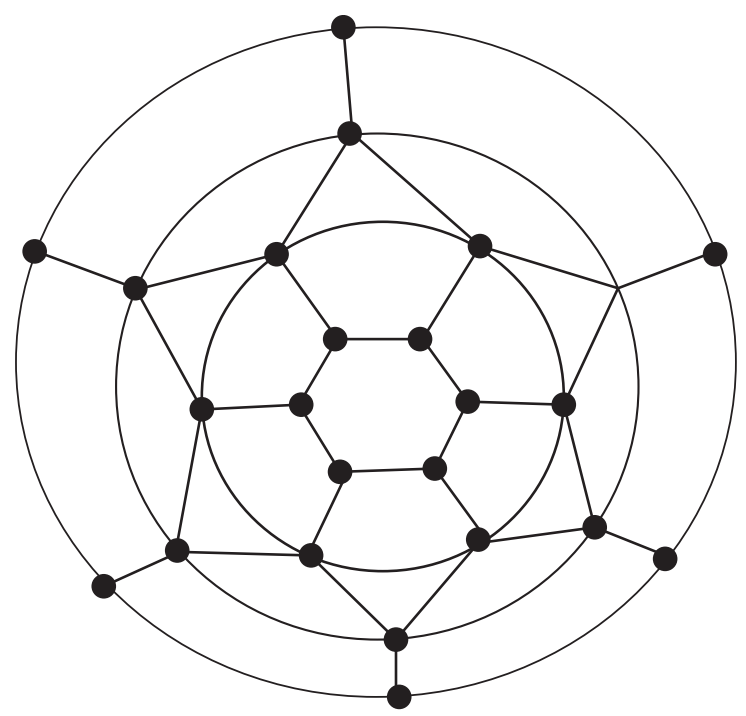

Figure 3: Graph of double antiprism $S_{6}$. 


$$
\begin{aligned}
J\left(S_{x}+S_{y}\right) g(x, y) & =\frac{4}{3} n x^{6}+n x^{9}+\frac{1}{2} n x^{8}+\frac{5}{6} n x^{10}+\frac{1}{3} n x^{12}, \\
Q_{-2} J\left(S_{x}+S_{y}\right) g(x, y) & =\frac{4}{3} n x^{4}+n x^{7}+\frac{1}{2} n x^{6}+\frac{5}{6} n x^{8}+\frac{1}{3} n x^{10}, \\
S_{x} Q_{-2} J\left(S_{x}+S_{y}\right) g(x, y) & =\frac{1}{3} n x^{4}+\frac{n}{7} x^{7}+\frac{1}{12} n x^{6}+\frac{5}{48} n x^{8}+\frac{1}{30} n x^{10}, \\
2 S_{x} Q_{-2} J\left(L_{x}+L_{y}\right) g(x, y) & =\frac{8}{7} n x^{7}+\frac{4}{5} n x^{10}+\frac{1}{3} n x^{12}+\frac{4}{13} n x^{13}+\frac{2}{7} n x^{14}+\frac{1}{4} n x^{16} .
\end{aligned}
$$

Hence, we conclude the following results:

(1) $A B C\left[T_{n}\right]=D_{x}^{1 / 2} Q_{-2} J S_{x}^{1 / 2} S_{y}^{1 / 2} \quad[g(x, y)]_{x=1}=(n / 12)$ $(16+4 \sqrt{14}+3 \sqrt{6}+\sqrt{48}+\sqrt{10})$

(2) $G A\left[T_{n}\right]=2 S_{x} J D_{x}^{1 / 2} D_{y}^{1 / 2}[g(x, y)]_{x=1}=(n / 15)(30+$ $10 \sqrt{2}+6 \sqrt{6})$

(3) $B_{1}\left[T_{n}\right]=\left(D_{x}+D_{y}+2 D_{x} Q_{-2} J\right)[g(x, y)]_{x=y=1}=178 n$

(4) $B_{2}\left[T_{n}\right]=D_{x} Q_{-2} J\left(D_{x}+D_{y}\right)[g(x, y)]_{x=1}=502 n$

(5) $H B_{1}\left[T_{n}\right]=\left(D_{x}^{2}+D_{y}^{2}+2 D_{x}^{2} Q_{-2} J+2 D_{x} Q_{-2} J\left(D_{x}+\right.\right.$ $\left.\left.D_{y}\right)\right)[g(x, y)]_{x=y=1}=2126 n$

(6) $H B_{2}\left[T_{n}\right]=D_{x}^{2} Q_{-2} J\left(D_{x}^{2}+D_{y}^{2}\right)[g(x, y)]_{x=1}=19994 n$

(7) ${ }^{m} B_{1}\left[T_{n}\right]=S_{x} Q_{-2} J\left(L_{x}+L_{y}\right)[g(x, y)]_{x=1}=(17033 /$ 10920)n

(8) ${ }^{m} B_{2}\left[T_{n}\right]=S_{x} Q_{-2} J \quad\left(S_{x}+S_{y}\right)[g(x, y)]_{x=1}=(1171 /$ 1680)n

(9) $H_{b}\left[T_{n}\right]=2 S_{x} Q_{-2} J\left(L_{x}+L_{y}\right)[g(x, y)]_{x=1}=(17033 /$ 5460)n.

\section{M-Polynomial and Topological Indices of Convex Polytopes $A_{n}$}

Here, we will find the topological indices of $A_{n}$ using its $M$ polynomial.
Theorem 2. The M-polynomial of $A_{n}$ is

$$
M\left[\left(A_{n}\right) ; x ; y\right]=2 n x^{4} y^{4}+4 n x^{4} y^{6}+n x^{6} y^{6} .
$$

Then,

(1) $A B C\left[A_{n}\right]=(n / 36)(18 \sqrt{6}+48 \sqrt{3}+6 \sqrt{10})$

(2) $G A\left[A_{n}\right]=(n / 5)(15+8 \sqrt{6})$

(3) $B_{1}\left[A_{n}\right]=176 n$

(4) $B_{2}\left[A_{n}\right]=536 n$

(5) $H B_{1}\left[A_{n}\right]=2272 n$

(6) $H B_{2}\left[A_{n}\right]=2768 n$

(7) ${ }^{m} B_{1}\left[A_{n}\right]=(961 / 840) n$

(8) ${ }^{m} B_{2}\left[A_{n}\right]=(49 / 120) n$

(9) $H_{b}\left[A_{n}\right]=(961 / 420) n$.

$$
\begin{aligned}
D_{x} g(x ; y) & =8 n x^{4} y^{4}+16 n x^{4} y^{6}+6 n x^{6} y^{6} \\
D_{y} g(x ; y) & =8 n x^{4} y^{4}+24 n x^{4} y^{6}+6 n x^{6} y^{6} \\
\left(D_{x}+D_{y}\right)[g(x ; y)] & =16 n x^{4} y^{4}+40 n x^{4} y^{6}+12 n x^{6} y^{6} \\
S_{y}^{1 / 2} g(x, y) & =n x^{4} y^{4}+\frac{4}{\sqrt{6}} n x^{4} y^{6}+\frac{n}{\sqrt{6}} x^{6} y^{6} \\
S_{x}^{1 / 2} S_{y}^{1 / 2} g(x, y) & =\frac{n}{2} x^{4} y^{4}+\frac{4}{\sqrt{6}} n x^{4} y^{6}+\frac{n}{\sqrt{6}} x^{6} y^{6} \\
J S_{x}^{1 / 2} S_{y}^{1 / 2} g(x, y) & =\frac{n}{2} x^{8}+\frac{4}{\sqrt{6}} n x^{10}+\frac{n}{\sqrt{6}} x^{12} \\
Q_{-2} J S_{x}^{1 / 2} S_{y}^{1 / 2} g(x, y) & =\frac{n}{2} x^{6}+\frac{4}{\sqrt{6}} n x^{8}+\frac{n}{\sqrt{6}} x^{10} \\
D_{x}^{1 / 2} Q_{-2} J S_{x}^{1 / 2} S_{y}^{1 / 2} g(x, y) & =\sqrt{\frac{3}{2} n x^{6}+4} \sqrt{\frac{1}{3} n x^{8}+\frac{\sqrt{10}}{6} n x^{10}}
\end{aligned}
$$




$$
\begin{aligned}
& D_{y}^{1 / 2}[g(x, y)]=4 n x^{4} y^{4}+4 \sqrt{6} n x^{4} y^{6}+\sqrt{6} n x^{6} y^{6}, \\
& D_{x}^{1 / 2} D_{y}^{1 / 2}[g(x, y)]=8 n x^{4} y^{4}+8 \sqrt{6} n x^{4} y^{6}+6 n x^{6} y^{6} \text {, } \\
& J D_{x}^{1 / 2} D_{y}^{1 / 2}[g(x, y)]=8 n x^{8}+8 \sqrt{6} n x^{10}+6 n x^{12} \text {, } \\
& S_{x} J D_{x}^{1 / 2} D_{y}^{1 / 2}[g(x, y)]=n x^{8}+\frac{4}{5} \sqrt{6} n x^{10}+\frac{1}{2} n x^{12}, \\
& 2 S_{x} J D_{x}^{1 / 2} D_{y}^{1 / 2}[g(x, y)]=2 n x^{8}+\frac{8}{5} \sqrt{6} n x^{10}+n x^{12}, \\
& J_{g}(x ; y)=2 n x^{8}+4 n x^{10}+n x^{12} ; \\
& Q_{-2} J_{g}(x ; y)=2 n x^{6}+4 n x^{8}+n x^{10} ; \\
& D_{x} Q_{-2} J_{g}(x ; y)=12 n x^{6}+32 n x^{8}+10 n x^{10} \text {; } \\
& 2 D_{x} Q_{-2} J_{g}(x ; y)=24 n x^{6}+64 n x^{8}+20 n x^{10} \text {; } \\
& J\left(D_{x}+D_{y}\right) g(x ; y)=16 n x^{8}+40 n x^{10}+12 n x^{12} ; \\
& Q_{-2} J\left(D_{x}+D_{y}\right) g(x ; y)=16 n x^{6}+40 n x^{8}+12 n x^{10} \text {; } \\
& D_{x} Q_{-2} J\left(D_{x}+D_{y}\right) g(x ; y)=96 n x^{6}+320 n x^{8}+120 n x^{10} \text {; } \\
& D_{x}^{2} g(x ; y)=32 n x^{4} y^{4}+64 n x^{4} y^{6}+36 n x^{6} y^{6} \text {; } \\
& D_{y}^{2} g(x ; y)=32 n x^{4} y^{4}+144 n x^{4} y^{6}+36 n x^{6} y^{6} \text {; } \\
& \left(D_{x}^{2}+D_{y}^{2}\right) g(x ; y)=64 n x^{4} y^{4}+208 n x^{4} y^{6}+72 n x^{6} y^{6} ; \\
& D_{x}^{2} Q_{-2} J g(x ; y)=72 n x^{6}+256 n x^{8}+100 n x^{10} \text {; } \\
& 2 D_{x}^{2} Q_{-2} J g(x ; y)=144 n x^{6}+512 n x^{8}+200 n x^{10} \text {; } \\
& 2 D_{x} Q_{-2} J\left(D_{x}+D_{y}\right) g(x ; y)=192 n x^{6}+640 n x^{8}+240 n x^{10} \text {; } \\
& J\left(D_{x}^{2}+D_{y}^{2}\right) g(x ; y)=64 n x^{8}+208 n x^{10}+72 n x^{12} ; \\
& Q_{-2} J\left(D_{x}^{2}+D_{y}^{2}\right) g(x ; y)=64 n x^{6}+208 n x^{8}+72 n x^{10} ; \\
& D_{x}^{2} Q_{-2} J\left(D_{x}^{2}+D_{y}^{2}\right) g(x ; y)=384 n x^{6}+1664 n x^{8}+720 n x^{10} ; \\
& L_{x} g(x ; y)=2 n x^{8} y^{4}+4 n x^{8} y^{6}+n x^{12} y^{6} ; \\
& L_{y} g(x ; y)=2 n x^{4} y^{8}+4 n x^{4} y^{12}+n x^{6} y^{12} \\
& J\left(L_{x}+L_{y}\right) g(x ; y)=4 n x^{12}+4 n x^{16}+4 n x^{14}+2 n x^{18} ; \\
& Q_{-2} J\left(L_{x}+L_{y}\right) g(x ; y)=4 n x^{10}+4 n x^{14}+4 n x^{12}+2 n x^{16} ; \\
& S_{x} Q_{-2} J\left(L_{x}+L_{y}\right) g(x, y)=\frac{2}{5} n x^{10}+\frac{2}{7} n x^{14}+\frac{1}{3} n x^{12}+\frac{1}{8} n x^{16} \text {, } \\
& S_{y} g(x, y)=\frac{1}{2} n x^{4} y^{4}+\frac{2}{3} n x^{4} y^{6}+\frac{n}{6} n x^{6} y^{6}, \\
& S_{x} g(x, y)=\frac{1}{2} n x^{4} y^{4}+n x^{4} y^{6}+\frac{n}{6} n x^{6} y^{6} \text {, } \\
& \left(S_{x}+S_{y}\right) g(x, y)=n x^{4} y^{4}+\frac{5}{3} n x^{4} y^{6}+\frac{n}{3} n x^{6} y^{6}, \\
& J\left(S_{x}+S_{y}\right) g(x, y)=n x^{8}+\frac{5}{3} n x^{10}+\frac{n}{3} n x^{12}, \\
& Q_{-2} J\left(S_{x}+S_{y}\right) g(x, y)=n x^{6}+\frac{5}{3} n x^{8}+\frac{n}{3} n x^{10}, \\
& S_{x} Q_{-2} J\left(S_{x}+S_{y}\right) g(x, y)=\frac{n}{6} x^{6}+\frac{5}{24} n x^{8}+\frac{n}{30} n x^{10}, \\
& 2 S_{x} Q_{-2} J\left(L_{x}+L_{y}\right) g(x, y)=\frac{4}{5} n x^{10}+\frac{4}{7} n x^{14}+\frac{2}{3} n x^{12}+\frac{1}{4} n x^{16} \text {. }
\end{aligned}
$$


Hence, we conclude the following results:

(1) $A B C\left[A_{n}\right]=D_{x}^{1 / 2} Q_{-2} J S_{x}^{1 / 2} S_{y}^{1 / 2} \quad[g(x, y)]_{x=1}=(n / 36)$ $(18 \sqrt{6})+48 \sqrt{3}+6 \sqrt{10}$

(2) $G A\left[A_{n}\right]=2 S_{x} J D_{x}^{1 / 2} D_{y}^{1 / 2} \quad[g(x, y)]_{x=1}=(n / 5)(15+$ $8 \sqrt{6})$

(3) $B_{1}\left[A_{n}\right]=\left(D_{x}+D_{y}+2 D_{x} Q_{-2} J\right)[g(x, y)]_{x=y=1}=176 n$

(4) $B_{2}\left[A_{n}\right]=D_{x} Q_{-2} J\left(D_{x}+D_{y}\right)[g(x, y)]_{x=1}=536 n$

(5) $H B_{1}\left[A_{n}\right]=\left(D_{x}^{2}+D_{y}^{2}+2 D_{x}^{2} Q_{-2} J+2 D_{x} Q_{-2} J \quad\left(D_{x}+\right.\right.$ $\left.\left.D_{y}\right)\right)[g(x, y)]_{x=y=1}=2272 n$

(6) $H B_{2}\left[A_{n}\right]=D_{x}^{2} Q_{-2} J\left(D_{x}^{2}+D_{y}^{2}\right)[g(x, y)]_{x=1}=2768 n$

(7) ${ }^{m} B_{1}\left[A_{n}\right]=S_{x} Q_{-2} J\left(L_{x}+L_{y}\right) \quad[g(x, y)]_{x=1}=(961 /$ $840) n$

(8) ${ }^{m} B_{2}\left[A_{n}\right]=S_{x} Q_{-2} J\left(S_{x}+S_{y}\right)[g(x, y)]_{x=1}=(49 / 120) n$

(9) $H_{b}\left[A_{n}\right]=2 S_{x} Q_{-2} J \quad\left(L_{x}+L_{y}\right) \quad[g(x, y)]_{x=1}=(961 /$ 420)n.

\section{M-Polynomial and Topological Indices of Convex Polytopes $S_{n}$}

Now we will find the topological indices of $S_{n}$ using its $M$ polynomial.
Theorem 3. The M-polynomial of $S_{n}$ is

$$
M\left[S_{n} ; x ; y\right]=2 n x^{3} y^{3}+2 n x^{3} y^{5}+4 n x^{5} y^{5} .
$$

Then,

(1) $A B C\left[S_{n}\right]=(n / 15)(20+6 \sqrt{10}+24 \sqrt{2})$

(2) $G A\left[S_{n}\right]=(n / 2)(12+\sqrt{15})$

(3) $B_{1}\left[S_{n}\right]=172 n$

(4) $B_{2}\left[S_{n}\right]=464 n$

(5) $H B_{1}\left[S_{n}\right]=15824 n$

(6) $\mathrm{HB}_{2}\left[S_{n}\right]=1952$

(7) ${ }^{m} B_{1}\left[S_{n}\right]=(14332 / 9009)$

(8) ${ }^{m} B_{2}\left[S_{n}\right]=(32 / 45) n$

(9) $H_{b}\left[S_{n}\right]=(28664 / 9009) n$.

Proof

$$
\begin{aligned}
D_{x} g(x, y) & =6 n x^{3} y^{3}+6 n x^{3} y^{5}+20 n x^{5} y^{5}, \\
D_{y} g(x, y) & =6 n x^{3} y^{3}+10 n x^{3} y^{5}+20 n x^{5} y^{5}, \\
\left(D_{x}+D_{y}\right)[g(x, y)] & =12 n x^{3} y^{3}+16 n x^{3} y^{5}+40 n x^{5} y^{5}, \\
S_{y}^{1 / 2} g(x, y) & =\frac{2}{\sqrt{3}} n x^{3} y^{3}+\frac{2}{\sqrt{5}} n x^{3} y^{5}+\frac{4}{\sqrt{5}} n x^{5} y^{5}, \\
S_{x}^{1 / 2} S_{y}^{1 / 2} g(x, y) & =\frac{2}{3} n x^{3} y^{3}+\frac{2}{\sqrt{15}} n x^{3} y^{5}+\frac{4}{5} n x^{5} y^{5}, \\
J S_{x}^{1 / 2} S_{y}^{1 / 2} g(x, y) & =\frac{2}{3} n x^{6}+\frac{2}{\sqrt{15}} n x^{8}+\frac{4}{5} n x^{10}, \\
Q_{-2} J S_{x}^{1 / 2} S_{y}^{1 / 2} g(x, y) & =\frac{2}{3} n x^{4}+\frac{2}{\sqrt{15}} n x^{6}+\frac{4}{5} n x^{8}, \\
D_{x}^{1 / 2} Q_{-2} J S_{x}^{1 / 2} S_{y}^{1 / 2} g(x, y) & =\frac{4}{3} n x^{4}+\frac{2 \sqrt{6}}{\sqrt{15}} n x^{6}+\frac{8 \sqrt{2}}{5} n x^{8}, \\
S_{x} J D_{x}^{1 / 2} D_{y}^{1 / 2}[g(x, y)] & =n x^{6}+\frac{\sqrt{15}}{4} n x^{8}+2 n x^{10}, \\
J S_{x} J D_{x}^{1 / 2} D_{y}^{1 / 2}[g(x, y)] & =2 n x^{6}+\frac{\sqrt{15}}{2} n x^{8}+4 n x^{10}, \\
D_{x}^{1 / 2} D_{y}^{1 / 2}[g(x, y)] & =2 \sqrt{3} n x^{3} y^{3}+2 \sqrt{5} n x^{3} y^{5}+4 \sqrt{5} n x^{5} y^{5}, \\
& =6 n x^{3} y^{3}+2 \sqrt{15} n x^{3} y^{5}+20 n x^{5} y^{5}, \\
&
\end{aligned}
$$




$$
\begin{aligned}
& J g(x, y)=2 n x^{6}+2 n x^{8}+4 n x^{10}, \\
& Q_{-2} J g(x, y)=2 n x^{4}+2 n x^{6}+4 n x^{8}, \\
& D_{x} Q_{-2} J g(x, y)=8 n x^{4}+12 n x^{6}+32 n x^{8} \text {, } \\
& 2 D_{x} Q_{-2} J g(x, y)=16 n x^{4}+24 n x^{6}+64 n x^{8} \text {, } \\
& J\left(D_{x}+D_{y}\right) g(x, y)=12 n x^{6}+16 n x^{8}+40 n x^{10}, \\
& Q_{-2} J\left(D_{x}+D_{y}\right) g(x, y)=12 n x^{4}+16 n x^{6}+40 n x^{8}, \\
& D_{x} Q_{-2} J\left(D_{x}+D_{y}\right) g(x, y)=48 n x^{4}+96 n x^{6}+320 n x^{8} \text {, } \\
& D_{x}^{2} g(x, y)=18 n x^{3} y^{3}+18 n x^{3} y^{5}+100 n x^{5} y^{5}, \\
& D_{y}^{2} g(x, y)=18 n x^{3} y^{3}+50 n x^{3} y^{5}+100 n x^{5} y^{5} \text {, } \\
& \left(D_{x}^{2}+D_{y}^{2}\right) g(x, y)=36 n x^{3} y^{3}+68 n x^{3} y^{5}+200 n x^{5} y^{5} \text {, } \\
& D_{x}^{2} Q_{-2} J g(x, y)=32 n x^{4}+72 n x^{6}+256 n x^{8} \text {, } \\
& 2 D_{x}^{2} Q_{-2} J g(x, y)=64 n x^{4}+144 n x^{6}+512 n x^{8}, \\
& 2 D_{x} Q_{-2} J\left(D_{x}+D_{y}\right) g(x, y)=96 n x^{4}+192 n x^{6}+640 n x^{8} \text {, } \\
& J\left(D_{x}^{2}+D_{y}^{2}\right) g(x, y)=36 n x^{6}+68 n x^{8}+200 n x^{10}, \\
& Q_{-2} J\left(D_{x}^{2}+D_{y}^{2}\right) g(x, y)=36 n x^{4}+68 n x^{6}+200 n x^{8}, \\
& D_{x}^{2} Q_{-2} J\left(D_{x}^{2}+D_{y}^{2}\right) g(x, y)=576 n x^{4}+2448 n x^{6}+12800 n x^{8} \text {, } \\
& L_{x} g(x, y)=2 n x^{6} y^{3}+2 n x^{6} y^{5}+4 n x^{10} y^{5}, \\
& L_{y} g(x, y)=2 n x^{3} y^{6}+2 n x^{3} y^{10}+4 n x^{5} y^{10}, \\
& J\left(L_{x}+L_{y}\right) g(x, y)=4 n x^{9}+8 n x^{15}+2 n x^{13}+2 n x^{11}, \\
& Q_{-2} J\left(L_{x}+L_{y}\right) g(x, y)=4 n x^{7}+8 n x^{13}+2 n x^{11}+2 n x^{9}, \\
& S_{x} Q_{-2} J\left(L_{x}+L_{y}\right) g(x, y)=\frac{4}{7} n x^{7}+\frac{8}{13} n x^{13}+\frac{2}{11} n x^{11}+\frac{2}{9} n x^{9} \text {, } \\
& S_{y} g(x, y)=\frac{1}{2} n x^{4} y^{4}+\frac{2}{3} n x^{4} y^{6}+\frac{n}{6} x^{6} y^{6}, \\
& S_{x} g(x, y)=\frac{1}{2} n x^{4} y^{4}+n x^{4} y^{6}+\frac{n}{6} x^{6} y^{6}, \\
& \left(S_{x}+S_{y}\right) g(x, y)=n x^{4} y^{4}+\frac{5}{3} n x^{4} y^{6}+\frac{n}{3} x^{6} y^{6}, \\
& J\left(S_{x}+S_{y}\right) g(x, y)=n x^{8}+\frac{5}{3} n x^{10}+\frac{n}{3} x^{12}, \\
& Q_{-2} J\left(S_{x}+S_{y}\right) g(x, y)=n x^{6}+\frac{5}{3} n x^{8}+\frac{n}{3} x^{10}, \\
& S_{x} Q_{-2} J\left(S_{x}+S_{y}\right) g(x, y)=\frac{n}{6} x^{6}+\frac{5}{24} n x^{8}+\frac{n}{30} x^{10}, \\
& 2 S_{x} Q_{-2} J\left(L_{x}+L_{y}\right) g(x, y)=\frac{8}{7} n x^{7}+\frac{16}{13} n x^{13}+\frac{4}{11} n x^{11}+\frac{4}{9} n x^{9} \text {. }
\end{aligned}
$$

Hence, we conclude the following results:
(1) $A B C\left[S_{n}\right]=D_{x}^{1 / 2} Q_{-2} J S_{x}^{1 / 2} S_{y}^{1 / 2} \quad[g(x, y)]_{x=1}=(n / 15)$ $(20+6 \sqrt{10}+24 \sqrt{2})$
(2) $G A\left[S_{n}\right]=2 S_{x} J D_{x}^{1 / 2} D_{y}^{1 / 2}[g(x, y)]_{x=1}=(n / 2)(12+\sqrt{15})$
(3) $B_{1}\left[S_{n}\right]=\left(D_{x}+D_{y}+2 D_{x} Q_{-2} J\right) \quad[g(x, y)]_{x=y=1}=$ $172 n$
(4) $B_{2}\left[S_{n}\right]=D_{x} Q_{-2} J\left(D_{x}+D_{y}\right)[g(x, y)]_{x=1}=464 n$

(5) $H B_{1}\left[S_{n}\right]=\left(D_{x}^{2}+D_{y}^{2}+2 D_{x}^{2} Q_{-2} J+2 D_{x} Q_{-2} J \quad\left(D_{x}+\right.\right.$ $\left.\left.D_{y}\right)\right)[g(x, y)]_{x=y=1}=15824 n$

(6) $H B_{2}\left[S_{n}\right]=D_{x}^{2} Q_{-2} J\left(D_{x}^{2}+D_{y}^{2}\right)[g(x, y)]_{x=1}=1952 n$

(7) ${ }^{m} B_{1}\left[S_{n}\right]=S_{x} Q_{-2} J\left(L_{x}+L_{y}\right) \quad[g(x, y)]_{x=1}=(14332 /$ 9009)n

(8) ${ }^{m} B_{1}\left[S_{n}\right]=S_{x} Q_{-2} J\left(S_{x}+S_{y}\right)[g(x, y)]_{x=1}=(32 / 45) n$

(9) $H_{b}\left[S_{n}\right]=2 S_{x} Q_{-2} J\left(L_{x}+L_{y}\right)[g(x, y)]_{x=1}=(28664 /$ 9009)n. 


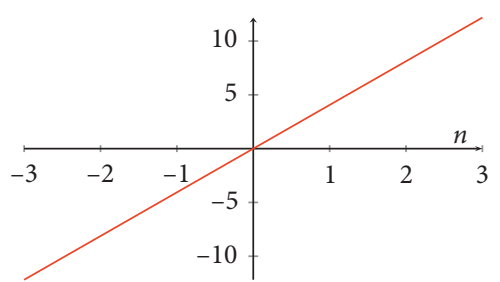

(a)

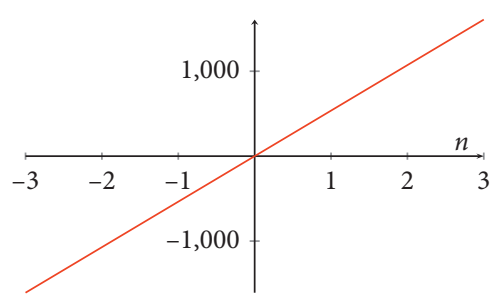

(d)

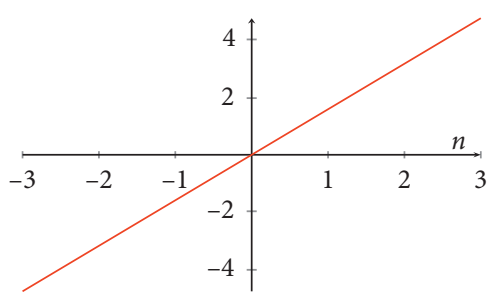

(g)

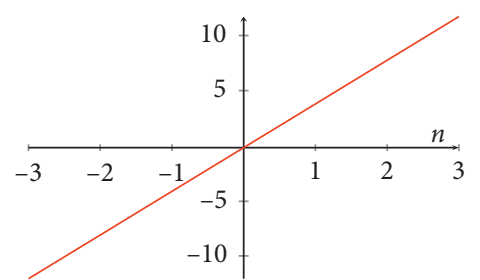

(b)

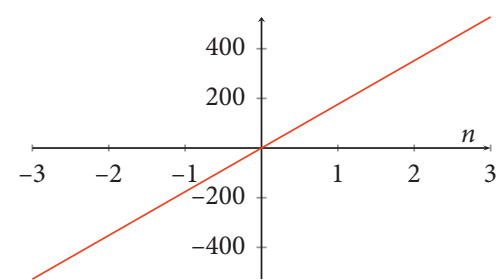

(c)

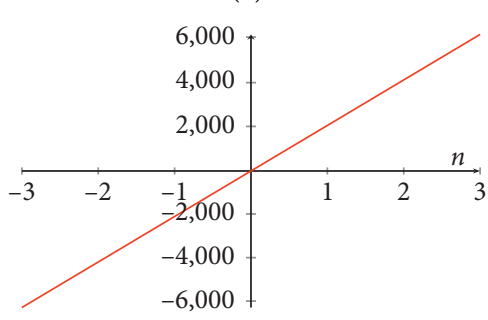

(e)

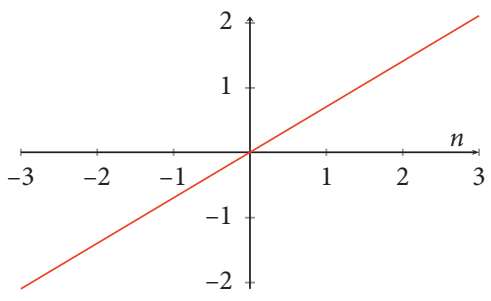

(h)

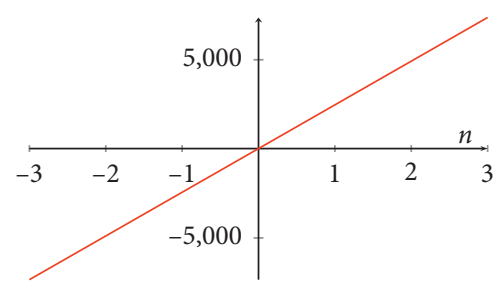

(f)

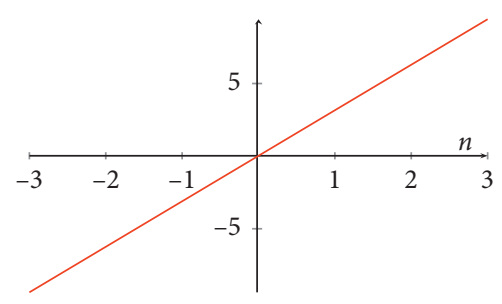

(i)

Figure 4: The plot of topological indices of convex polytope $T_{n}$. (a) Atom-bond connectivity index, (b) geometric arithmetic index, (c) first $K$-Banhatti index, (d) second $K$-Banhatti index, (e) first $K$-hyper-Banhatti index, (f) second $K$-hyper-Banhatti index, (g) modified first $K$ Banhatti index, (h) modified second $K$-Banhatti index, and (i) harmonic $K$-Banhatti index.

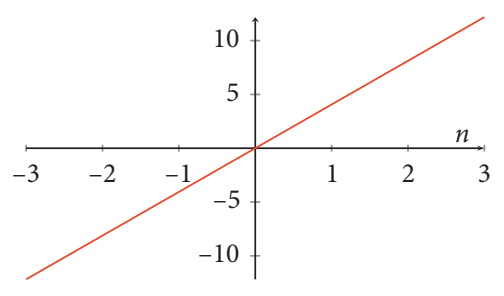

(a)

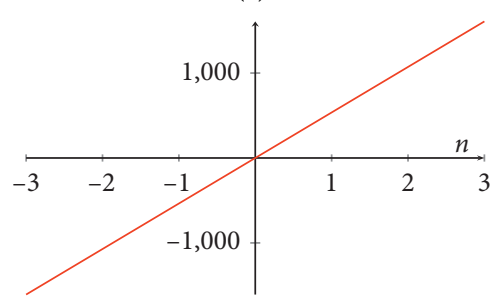

(d)

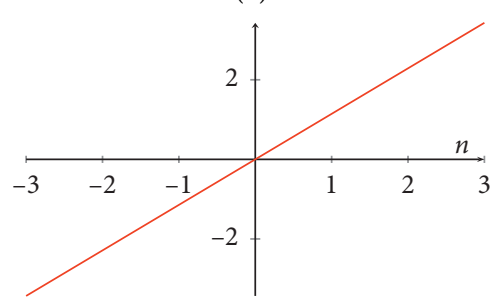

(g)

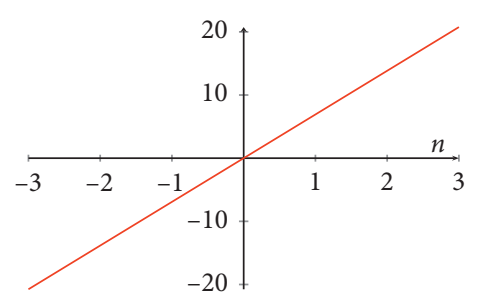

(b)

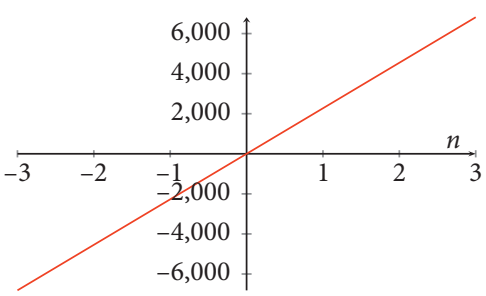

(e)

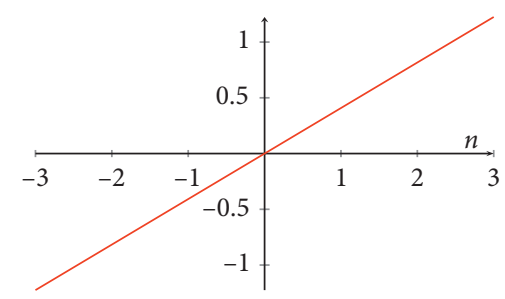

(h)

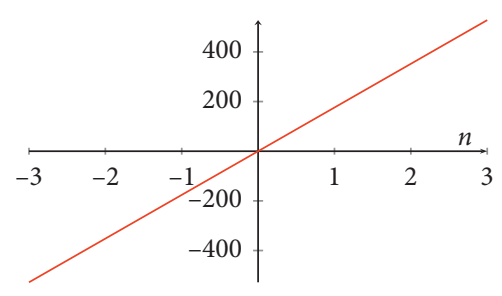

(c)

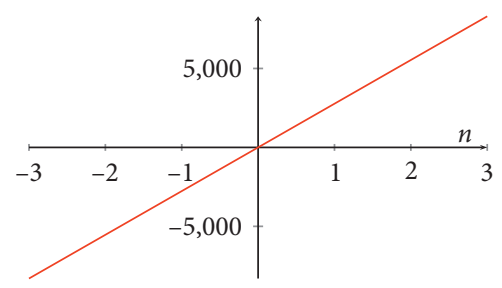

(f)

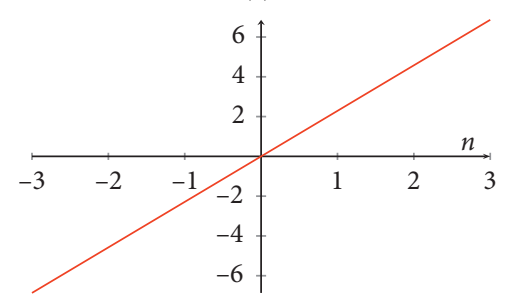

(i)

Figure 5: The plot of topological indices of convex polytope $A_{n}$. (a) Atom-bond connectivity index, (b) geometric arithmetic index, (c) first $K$-Banhatti index, (d) second $K$-Banhatti index, (e) first $K$-hyper-Banhatti index, (f) second $K$-hyper-Banhatti index, (g) modified first $K$ Banhatti index, (h) modified second $K$-Banhatti index, and (i) harmonic $K$-Banhatti index. 


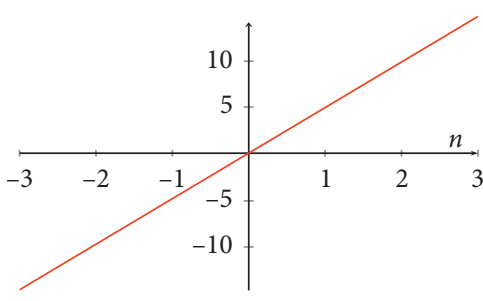

(a)

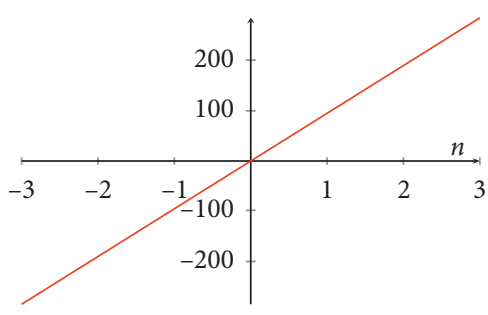

(d)

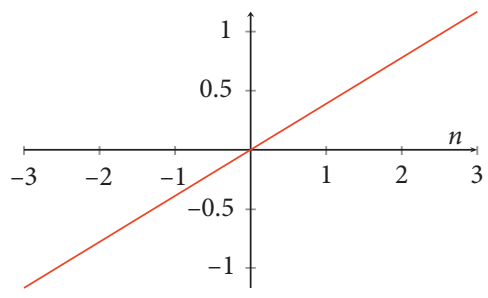

(g)

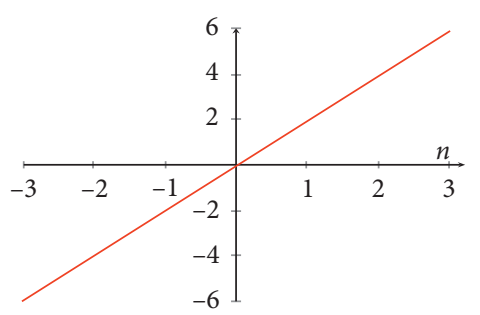

(b)

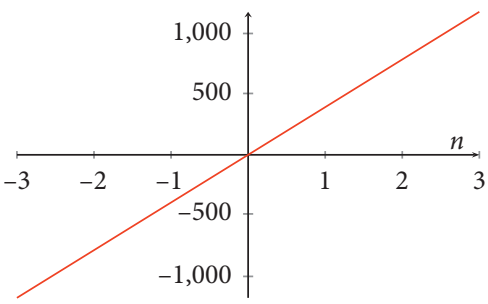

(e)

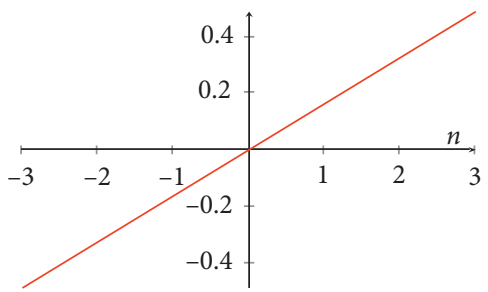

(h)

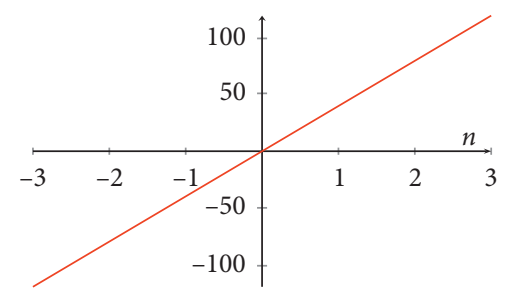

(c)

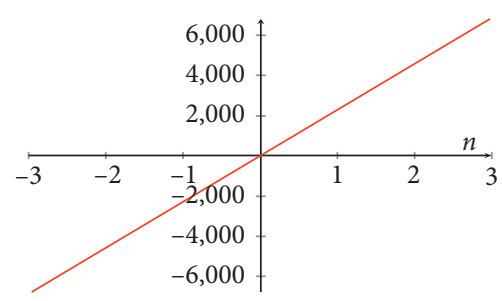

(f)

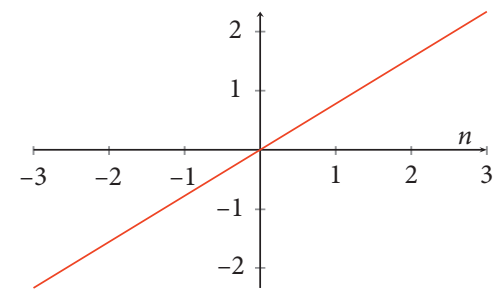

(i)

Figure 6: The plot of topological indices of convex polytope $S_{n}$. (a) Atom-bond connectivity index, (b) geometric arithmetic index, (c) first $K$-Banhatti index, (d) second $K$-Banhatti index, (e) first $K$-hyper-Banhatti index, (f) second $K$-hyper-Banhatti index, (g) modified first $K$ Banhatti index, (h) modified second $K$-Banhatti index, and (i) harmonic $K$-Banhatti index.

TABLE 1: Topological indices derived from $M(G ; x ; y)=$ $g(x ; y)[12]$.

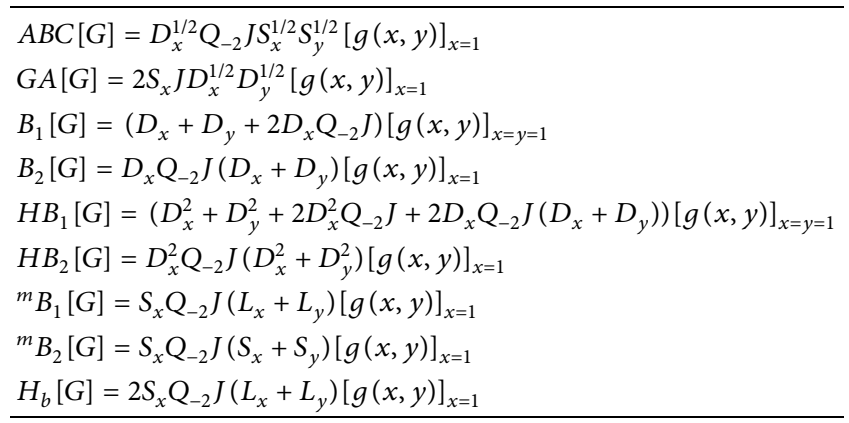

\section{Graphical Analysis}

Graphs are helpful as they elucidate the facts in visual form. Moreover, we demonstrate the behavior of $M$-polynomial and topological indices by drawing graphics. A graph stores information of the chemical structures that is equal to several words. The information that could not be represented in terms of words can be clarified in a better way by means of a graph. This graphical description provides us a great way to understand results against parameters (see Figures 4-6 ).

\section{Conclusion}

Topological indices are a numerical quantity computed from the graph of any compound and typically remain the same under graph isomorphism. These are used to predict and model certain properties of different compounds. We can the easily understand the properties, for example, the chemical reactivity, biological activities, and physical features of associated molecules. In this work, we have calculated very important degree-based topological indices such as atom-bond connectivity index, geometric arithmetic index, $K$-Banhatti indices, $K$-hyper-Banhatti indices and modified $K$-Banhatti indices of some families of cycle graphs which are given in Table 1 with the help of their $M$-polynomials. These findings can play a huge role in industry and pharmacy because these indices are absolutely functions of chemical graphs and explain many chemical properties as viscosity, strain energy, and heat of formation etc.

\section{Data Availability}

No data were used to support this study.

\section{Conflicts of Interest}

The authors declare no conflicts of interest. 


\section{References}

[1] H. Wiener, "Structural determination of paraffin boiling points," Journal of the American Chemical Society, vol. 69, no. 1, pp. 17-20, Article ID 20291038, 1947.

[2] S. M. Kang, W. Nazeer, W. Gao, D. Afzal, and S. N. Gillani, "M-polynomials and topological indices of dominating David derived networks," Open Chemistry, vol. 16, no. 1, pp. 201-213, 2018.

[3] V. Kulli, "On K-Banhatti indices of graphs," Journal of Computer and Mathematical Sciences, vol. 7, no. 4, pp. 213218, 2016.

[4] E. Estrada, L. Torres, L. Rodriguez, and I. Gutman, "An atombond connectivity index: modelling the enthalpy of formation of alkanes," Indian Journal of Chemistry, vol. 37, no. 10, pp. 849-855, 1998.

[5] F. Afzal, S. Hussain, D. Afzal, S. Hameed, and S. Hameed, " $M-$ polynomial and topological indices of zigzag edge coronoid fused by starphene," Open Chemistry, vol. 18, no. 1, pp. 1362-1369, 2020.

[6] M. Cancan, S. Ediz, H. Mutee-Ur-Rehman, and D. Afzal, " $M$ polynomial and topological indices poly (EThyleneAmidoAmine) dendrimers," Journal of Information and Optimization Sciences, vol. 41, no. 4, pp. 1117-1131, 2020.

[7] S. Hussain, F. Afzal, D. Afzal, M. R. Farahani, M. Cancan, and S. Ediz, "Theoretical study of benzene ring embedded in Ptype surface in $2 \mathrm{~d}$ network using some new degree based topological indices via M-polynomial," Eurasian Chemical Communications, vol. 3, pp. 180-186, 2021.

[8] D. B. West, Introduction of Graph Theory, Prentice-Hall, Hoboken, NJ, USA, 2001.

[9] V. Kulli, "On K-hyper Banhatti indices and coindices of graphs," International Research Journal of Pure Algebra, vol. 6, no. 5, pp. 300-304, 2016.

[10] V. Kulli, "New K-Banhatti topological indices," International Journal of Fuzzy Mathematical Archive, vol. 12, no. 1, pp. 29-37, 2017.

[11] D. Vukičević and B. Furtula, "Topological index based on the ratios of geometrical and arithmetical means of end-vertex degrees of edges," Journal of Mathematical Chemistry, vol. 46, no. 4, pp. 1369-1376, 2009.

[12] F. Afzal, S. Hussain, D. Afzal, and S. Razaq, "Some new degree based topological indices via M-polynomial," Journal of Information and Optimization Sciences, vol. 41, no. 4, pp. 1061-1076, 2020. 